\title{
ANALYSIS OF MARKOV HM-QUEUEING NETWORKS WITH FIFO DISCIPLINE AT THE TRANSIENT REGIME AND ITS APPLICATION
}

\author{
Mikhail Matalytski \\ Institute of Mathematics, Czestochowa University of Technology \\ Częstochowa, Poland \\ michal.matalytski@im.pcz.pl
}

\begin{abstract}
In the paper Markov Queueing Networks (QN) are considered with the same types and different types of customers and incomes, FIFO discipline, which are probabilistic models of different Information Nets and Systems (INS). The incomes from the state transition of the network depend on servicing times of customers in the Queueing Systems (QS). The purpose of the research are design and development of methods and techniques of finding the probability-cost characteristics in such QN as effective analysis tools of INS. A closed Markov HM-network with the same types of customers has been investigated. Approximate expressions for the expected incomes of the QS were obtained. The method of finding the mean number of the customers was proposed. The analysis of an open HM-network with different types of customers and many-server queues has been carried out in the second part of the paper. Customers during the transition between QS can change its type. Approximate expressions for the expected incomes of the QS for each type of customer have been also obtained. A method for finding the mean number of servicing lines was described.
\end{abstract}

Keywords: HM-queueing network, closed and open queueing network, same types of customers and different types of customers, many-server queues, mean number of customers

\section{Introduction}

When analyzing the INS one of the important tasks is to assess the cost of income that they get from the operation of their various subsystems. This resulted in the appearance of new mathematical models $-\mathrm{QN}$ with incomes. These models differ from the classical that it is required in addition to studying random processes of customer servicing and take into account incomes and expenses. An important task in this case is the estimation and forecasting of the expected incomes in network systems.

In this paper the analysis of a closed Markov HM-network with the same types of customers has been carried out. It is assumed that the incomes from the transi- 
tions between the states of the network are random variables (RV) with timedependent customer servicing in the systems. Approximate expressions for the expected incomes has been obtained. A method of finding the mean number of the customers in the network system has been described. In the case, when the incomes from the transitions between the states of the network are random variables with given mean values, a method for finding the expected incomes of the HM-network systems with many-server queues and different types of customers has been proposed.

\section{Expected incomes of a closed Markov HM-networks with the same types of customers}

Let us consider a network with a central system of customer service, see [1]. The state of the network at time $t$ meaning the vector $k=\left(k_{1}, k_{2}, \ldots, k_{n}, t\right)$, where $k_{i}$ is the count of customers in the system $S_{i}, i=\overline{1, n}$.

We consider the case when the incomes from the transitions between the states of the network are functions that depend on the RV, which means the time of customers service in the central system $S_{n}$. In practice, this corresponds to the fact that the user can be the Internet for a random time. We obtain approximate expressions for the expected incomes of the system $S_{n}$.

Consider the dynamics of income changes of a network system $S_{n}$. Denote by the $V_{n}(t)$ its income at moment time $t$. Let the initial moment time income of the system equal $v_{n 0}$. The income of its QS at moment time $t+\Delta t$ can be represented in the form

$$
V_{n}(t+\Delta t)=V_{n}(t)+\Delta V_{n}(t, \Delta t)
$$

where $\Delta V_{n}(t, \Delta t)$ - income changes of the system $S_{n}$ at the time interval $[t, t+\Delta t)$. Denote by the $\xi-\mathrm{RV}$ with the distribution function (DF) $F_{\xi}(t)=1-e^{-\mu_{n}\left(k_{n}(t)\right) t}$, where $\xi$ - time of customers service in QS $S_{n}, R_{n i}(t)$ - some measurable functions, $i=\overline{1, n-1}$. Then $R_{n i}(\xi)$ also be RV, $i=\overline{1, n-1}$. Let $\mu_{i}\left(k_{i}(t)\right)$ - service rate of the $S_{i}, i=\overline{1, n} ; p_{n i}$ be the transition probability for customer from the $S_{n}$ QS to the $S_{i} \mathrm{QS}, i=\overline{1, n-1}$. The following cases are possible:

- with probability $\mu_{i}\left(k_{i}(t)\right) \Delta t+o(\Delta t)$ to the central system a customer will arrive from the $i$-th QS, which will not bring an income to the $S_{n}$ QS, $i=\overline{1, n-1}$; 
- with probability $\mu_{n}\left(k_{n}(t)\right) p_{n i} \Delta t+o(\Delta t)$ a customer from the central system will come to the $i$-th QS (peripheral QS), and its income reduced by the $R_{n i}(\xi)$, and the income of the $S_{n}$ QS increased by this RV, $i=\overline{1, n-1}$;

- with probability $1-\sum_{i=1}^{n} \mu_{i}\left(k_{i}(t)\right) \Delta t$ on time interval $\Delta t$ there will be no change of system $S_{i}$ nothing is going to happen.

Then we obtain

$$
\Delta V_{n}(t, \Delta t)=\left\{\begin{array}{cl}
-R_{n i}(\xi) & \text { with probability } \mu_{n}\left(k_{n}(t)\right) p_{n i} \Delta t+o(\Delta t), i=\overline{1, n-1} \\
0 & \text { with probability } 1-\mu_{n}\left(k_{n}(t)\right) \Delta t+o(\Delta t), i=\overline{1, n-1}
\end{array}\right.
$$

Let $M\left\{R_{n i}(\xi)\right\}=\int_{0}^{\infty} R_{n i}(t) d F_{\xi}(t)=b_{n i}, i=\overline{1, n-1}$. Then for a fixed implementation of process $k(t)$ we have:

$$
M\left\{\Delta V_{n}(t, \Delta t) / k(t)\right\}=\sum_{i=1}^{n-1} b_{n i} \mu_{n}\left(k_{n}(t)\right) p_{n i} \Delta t+o(\Delta t) .
$$

Further assume that the system $S_{n}$ contains $m_{n}$ identical service lines. The service times for customers are distributed exponentially with the rate $\mu_{n}$ in each service line. We assume that the service rate is linearly dependent on their number, i.e.

$$
\mu_{n}\left(k_{n}(t)\right)=\left\{\begin{array}{ll}
\mu_{n} k_{n}(t), & k_{n}(t) \leq m_{n}, \\
\mu_{n} m_{n}, & k_{n}(t)>m_{n},
\end{array}=\mu_{n} \min \left(k_{n}(t), m_{n}\right) .\right.
$$

We also assume that averaging of expression $\mu_{n}\left(k_{n}(t)\right)$ gives $\mu_{n} \min \left(N_{n}(t), m_{n}\right)$. It should be noted that this is performed in cases: a) times of customers service in QS constant, it does not match our situation; b) $S_{n}$ QS operates under a low-traffic regime, i.e. $\forall t \quad k_{n}(t) \leq m_{n}$ or a heavy-traffic regime $\forall t$ $k_{i}(t)>m_{i}, i=\overline{1, n}$. Then

$$
M\left\{\Delta V_{n}(t, \Delta t)\right\}=\mu_{n} \min \left(N_{n}(t), m_{n}\right) \sum_{i=1}^{n-1} b_{n i} p_{n i} \Delta t+o(\Delta t) .
$$

We introduce the notation $v_{n}(t)=M\left\{V_{n}(t)\right\}$. Then for the expected income of the central QS from (1) we obtain 
$v_{n}(t+\Delta t)=v_{n}(t)+M\left\{\Delta V_{n}(t, \Delta t)\right\}=v_{n}(t)+\mu_{n} \min \left(N_{n}(t), m_{n}\right) \sum_{i=1}^{n-1} b_{n i} p_{n i} \Delta t+o(\Delta t)$,

from where, passing to the limit $\Delta t \rightarrow 0$, we will have

$\frac{d v_{n}(t)}{d t}=\mu_{n} \min \left(N_{n}(t), m_{n}\right) \sum_{i=1}^{n-1} b_{n i} p_{n i}$,

e.g.

$$
v_{n}(t)=v_{n 0}+\mu_{n} \sum_{i=1}^{n-1} b_{n i} p_{n i} \int_{t_{0}}^{t} \min \left(N_{n}(\tau), m_{n}\right) d \tau .
$$

To find the mean number of customers $N_{n}(t)$ we can apply advanced recurrent MVA method, see [1].

We describe another method of income finding of the central system. A situation which corresponds to the practical - this is when in the network QS $S_{1}, S_{2}, \ldots, S_{n}$ in mean bursts are not observed, i.e. $\min \left(N_{i}(t), m_{i}\right)=N_{i}(t), i=\overline{1, n}$. Then the system of differential equations (DE) for the $N_{i}(t), i=\overline{1, n}$, written in the form (it is obtained similarly as in [1,2]):

$$
\left\{\begin{array}{l}
\frac{d N_{i}(t)}{d t}=-\mu_{i} N_{i}(t)+\mu_{n} N_{n}(t) p_{n i}, \quad i=\overline{1, n-1} \\
N_{n}(t)=K-\sum_{i=1}^{n-1} N_{i}(t)
\end{array}\right.
$$

By the initial conditions $N_{i}(0), i=\overline{1, n}$. Expression for the expected income, according to (3) has the form

$$
v_{n}(t)=v_{n 0}+\mu_{n} \sum_{i=1}^{n-1} b_{n i} p_{n i} \int_{t_{0}}^{t} N_{n}(\tau) d \tau .
$$

\subsection{Example}

Let count of customers in the network be equal to $K=500$ and count of QS equal $n=K+1$. Income changes of the network shall be considered in the time period at 10 hours, $t \in[0, T], T=10$. Set at the initial time the mean number of customers in the system equals $N_{1}(0)=0, \quad N_{i}(0)=1, \quad i=\overline{1, n-1}$ and $N_{n}(0)=K-\sum_{i=1}^{n-1} N_{i}(0)=1$. Service rates equal $\mu_{i}=0.5, i=\overline{1.500}, \mu_{n}=n-1$. 
Transition probabilities equal $p_{n i}=\frac{1}{n-1}, p_{i n}=1, i=\overline{1, n-1}$ and $p_{n n}=0$. Service lines count equal $m_{i}=1, i=\overline{1, n-1}$ and $m_{n}=K+1$. Let $R_{n i}(\xi)=5000 \xi+0,5$. Let at the initial time $t_{0}=0$ income of the system $S_{n}$ be equal to $v_{n 0}=0$. Then

$$
\begin{gathered}
b_{n i}=\int_{0}^{\infty} R_{n i}(t) d F_{\xi}(t)=-\int_{0}^{T}(5000 t+0.5) d\left(1-e^{-\mu_{n} t}\right)= \\
=\frac{5000\left(1-e^{-10 \mu_{n}}\left(10 \mu_{n}+1\right)\right)+0.5 \mu_{n}}{\mu_{n}}= \\
=\frac{5000\left(1-e^{-10(n-1)}(10(n-1)+1)\right)+0.5(n-1)}{n-1} \approx 10.5 .
\end{gathered}
$$

Further, using the formula (3) in conjunction with recurrent method of finding the mean number of customers (MVA-method) in the network system an income change of the $S_{n}$ system at the time interval $[0,10]$ has been obtained by using math packet Wolfram Mathematica, Figure 1 (straight line). Solving in Mathematica package system of DE (4), the mean number of customers in QS $S_{n}$ was obtained in an analytical form. Income changes chart of QS $S_{n}$ on time interval [0, $10]$ in this case, considering (5), is presented in Figure 1 as dashed line.

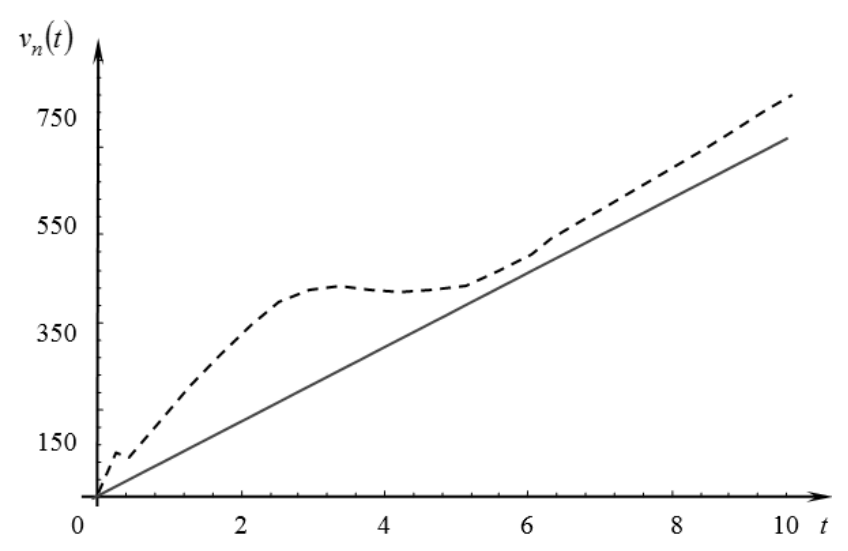

Fig. 1. Income changes of the QS $S_{n}$

\section{Research of an open HM-Network with different types of customers and many-server queues}

Network description with different types of customers is given in [2]. An issue of application was given of such a network in forecasting of incomes of service 
centers and points of information systems. We consider the case when the incomes from the state transition are RV or functions that depend on them. In the last case, the RV is the time of customer service in the QS.

Consider the dynamics of income changes of a network system $S_{i}, i=\overline{1, n}$. Let at the initial moment of time the income of this QS be equal to $v_{i 0}$. We are interested in income $V_{i}(t)$ at time $t$. The income of its QS at moment time $t+\Delta t$ can be represented in the form $V_{i}(t+\Delta t)=V_{i}(t)+\Delta V_{i}(t, \Delta t)$, where $\Delta V_{i}(t, \Delta t)$ - income changes of the system $S_{i}$ at the time interval $[t, t+\Delta t), i=\overline{1, n}$. Let $\xi_{i c}$ be the RV (time of customers service of type $c$ in the system $\left.S_{i}\right)$ with the DF $F_{\xi_{i c}}(t)=1-e^{-\mu_{i c} t}, i=\overline{1, n}$, $c=\overline{1, r}$. Denote by the $R_{i j}\left(\xi_{i c}\right)$ random income of the $S_{i}$ QS (really - losses) from the transition of customer of type $c$ from the system $S_{i}$ to the system $S_{j}, i=\overline{1, n}$, $c=\overline{1, d}$. Let $r_{i c}-\mathrm{RV}$ with a given expectation $\gamma_{i}^{(c)}$, meaning random income, which brings customer of type $c$ to the QS $S_{i}$ after finishing service, $i=\overline{1, n}$, $c=\overline{d+1, r}$.

To find the income of the system $S_{i}$ we write the conditional probabilities of the events that may occur during $\Delta t$. The following cases are possible:

a) With probability $q_{1}(i, c, \Delta t)=\lambda p_{0 c i c} \Delta t+o(\Delta t)$ from the external environment to the system $S_{i}$ will arrive a customer of type $c$, which will not bring an income;

b) With probability $q_{2}(i, c, t, \Delta t)=\mu_{i c} \varepsilon_{i c}(t) u\left(k_{i c}(t)\right) p_{i c 0 c} \Delta t+o(\Delta t)$, from the system $S_{i}$ a customer of type $c$ comes out from the network to the external environment, while the total amount of income of QS will not change, where $k_{i c}(t)$ customer count of type $c$ in the $i$-th QS at time $t, i=\overline{1, n}, c=\overline{1, r}$. If not all service lines are busy servicing customers of type $c$ inclusively. And there are some lines that are not busy servicing customers $\left(m_{i}>k_{i}(t)\right)$, or in the case when all the lines are busy servicing customers and queues are not observed $\left(m_{i}=k_{i}(t)\right)$, then value $\varepsilon_{i c}(t)$ takes a value equal to $\varepsilon_{i c}(t)=k_{i c}(t), i=\overline{1, n}$, $c=\overline{1, r}$;

c) A customer of type $c$ from the system $S_{i}$ goes into a $S_{j}$ as a customer of type $s$ with probability $q_{3}(i, c, j, s, t, \Delta t)=\mu_{i c} \varepsilon_{i c}(t) u\left(k_{i c}(t)\right) p_{i c j s} \Delta t+o(\Delta t), \quad j=\overline{1, n}$, $i \neq j, s=\overline{1, r}, c=\overline{1, d}$; in such a transition income of the system $S_{i}$ reduced by the value $R_{i j}\left(\xi_{i c}\right), j=\overline{1, n}, i \neq j, c=\overline{1, d}$.

For other types of customers income of the system $S_{i}$ reduced by the value $r_{i c}$ : in the case, if a customer of type $c$ from the system $S_{i}$ will come to the system $S_{j}$ 
as a customer of type $s$, with the same probability, $j=\overline{1, n}, j \neq i, s=\overline{1, r}$, $c=\overline{d+1, r}$.

d) With probability

$$
q_{4}(t, \Delta t)=1-\left(\lambda \sum_{i=1}^{n} \sum_{c=1}^{r} p_{0 c i c}+\sum_{i=1}^{n} \sum_{c=1}^{r} \sum_{j=0}^{n} \sum_{s=1}^{r} \mu_{i c} \varepsilon_{i c}(t) u\left(k_{i c}(t)\right) p_{i c j s}\right) \Delta t+o(\Delta t)
$$

at the time interval $\Delta t$ network state will not change.

Moreover, for each small time interval $\Delta t$ the system $S_{i}$ increases its income because of present therein customers of type $c$ by the value $\eta_{i c} \Delta t, i=\overline{1, n}, c=\overline{1, r}$. We assume, that RV $R_{i j}\left(\xi_{i c}\right), \eta_{i c}$ are pairwise independent, $i=\overline{1, n}, c=\overline{1, r}$.

Let $\Delta V_{i}^{(c)}(t, \Delta t)$ be the income changes of the system $S_{i}$ at the time interval $[t, t+\Delta t)$, connected with the transitions between QS of customers of type $c$. Then from the above it follows that

$$
\Delta V_{i}^{(c)}(t, \Delta t)=\left\{\begin{array}{l}
\eta_{i c} \Delta t \text { with probability } q_{1}(i, c, \Delta t)+q_{2}(i, c, t, \Delta t)+q_{4}(t, \Delta t), \\
-R_{i j}\left(\xi_{i c}\right)+\eta_{i c} \Delta t \quad \text { with probability } q_{3}(i, c, j, s, t, \Delta t), \\
j=\overline{1, n}, i \neq j, s=\overline{1, r}, c=\overline{1, d}, \\
-r_{i c}+\eta_{i c} \Delta t \quad \text { with probability } q_{3}(i, c, j, s, t, \Delta t), \\
j=\overline{1, n}, i \neq j, s=\overline{1, r}, c=\overline{d+1, r} .
\end{array}\right.
$$

We introduce the notation for the respective mathematical expectations: $M\left\{\eta_{i c}\right\}=\alpha_{i}^{(c)}, \quad M\left\{r_{i c}\right\}=\gamma_{i}^{(c)}, \quad c=\overline{d+1, r}, \quad \beta_{i j}^{(c)}=\mu_{i c} \int_{0}^{\infty} R_{i c}(t) e^{-\mu_{i c} t} d t, \quad i, j=\overline{1, n}$, $c=\overline{1, d}$. We find an expression for the expected income of the system $S_{i}$ at time $t$. For a fixed implementation of process $k(t)$ can be written:

$$
\begin{gathered}
M\left\{\Delta V_{i}^{(c)}(t, \Delta t) / k(t)\right\}= \\
=\alpha_{i}^{(c)}\left(q_{1}(i, c, \Delta t)+q_{2}(i, c, t, \Delta t)+q_{4}(t, \Delta t)+\sum_{j=1}^{n} \sum_{s=1}^{r} q_{3}(i, c, j, s, t, \Delta t)\right)- \\
-\sum_{j=1}^{n} \sum_{s=1}^{r}\left(u(d-c+1) \beta_{i j}^{(c)}+(1-u(d-c+1)) \gamma_{i}^{(c)}\right) q_{3}(i, c, j, s, t, \Delta t), i=\overline{1, n}, c=\overline{1, r} .
\end{gathered}
$$

Let $\varphi_{i j}^{(c)}=u(d-c+1) \beta_{i j}^{(c)}+(1-u(d-c+1)) \gamma_{i}^{(c)}, i=\overline{1, n}, c=\overline{1, r}$. Then (7) takes 
form

$$
\begin{gathered}
M\left\{\Delta V_{i}^{(c)}(t, \Delta t) / k(t)\right\}= \\
=\alpha_{i}^{(c)}\left(q_{1}(i, c, \Delta t)+q_{2}(i, c, t, \Delta t)+q_{4}(t, \Delta t)+\sum_{j=1}^{n} \sum_{s=1}^{r} q_{3}(i, c, j, s, t, \Delta t)\right)- \\
-\sum_{j=1}^{n} \sum_{s=1}^{r} \varphi_{i j}^{(c)} q_{3}(i, c, j, s, t, \Delta t), i=\overline{1, n}, c=\overline{1, r} .
\end{gathered}
$$

Further, substituting instead of functions $q_{1}(i, c, \Delta t), \quad q_{2}(i, c, t, \Delta t)$, $q_{3}(i, c, j, s, t, \Delta t)$ and $q_{4}(t, \Delta t)$ appropriately transition probabilities for customers of type $c$, we obtain

$$
\begin{gathered}
M\left\{\Delta V_{i}^{(c)}(t, \Delta t) / k(t)\right\}=\left(\alpha _ { i } ^ { ( c ) } \left(\lambda p_{0 c i c}+\mu_{i c} \varepsilon_{i c}(t) u\left(k_{i c}(t)\right) p_{i c 0 c}+1-\right.\right. \\
\left.-\left(\lambda \sum_{i=1}^{n} \sum_{c=1}^{r} p_{0 c i c}+\sum_{i=1}^{n} \sum_{c=1}^{r} \sum_{j=0}^{n} \sum_{s=1}^{r} \mu_{i c} \varepsilon_{i c}(t) u\left(k_{i c}(t)\right) p_{i c j s}\right)\right)- \\
\left.-\mu_{i c} \varepsilon_{i c}(t) u\left(k_{i c}(t)\right) \sum_{j=1}^{n} \varphi_{i j}^{(c)} \sum_{s=1}^{r} p_{i c j s}\right) \Delta t+o(\Delta t), i=\overline{1, n}, c=\overline{1, r} .
\end{gathered}
$$

As the expectation of the value $\varepsilon_{i c}(t) u\left(k_{i c}(t)\right)$ can take $M\left\{\varepsilon_{i c}(t) u\left(k_{i c}(t)\right)\right\}=\rho_{i c}(t)$, where $\rho_{i c}(t)$ - the mean number of busy service lines in the system $S_{i}$ by the customers of type $c$ at time $t, i=\overline{1, n}, c=\overline{1, r}$. So, averaging (8) by $k(t)$, we obtain

$$
M\left\{V_{i}^{(c)}(t, \Delta t)\right\}=f(i, c, t) \Delta t+o(\Delta t), i=\overline{1, n}, c=\overline{1, r},
$$

where

$$
\begin{gathered}
f(i, c, t)=\alpha_{i}^{(c)}\left(\lambda\left(p_{0 c i c}-1\right)+1\right)+\mu_{i c}\left(\alpha_{i}^{(c)} p_{i c 0 c}-\sum_{j=1}^{n} \sum_{s=1}^{r} \varphi_{i j}^{(c)} p_{i c j s}\right) \rho_{i c}(t)- \\
-\sum_{i=1}^{n} \sum_{j=0}^{n} \sum_{c, s=1}^{r} \mu_{i c} p_{i c j s} \rho_{i c}(t), i=\overline{1, n}, c=\overline{1, r}
\end{gathered}
$$

Then for the expected income $v_{i c}(t)=M\left\{V_{i}^{(c)}(t)\right\}$ of the system $S_{i}$, connected with the transitions between QS of customers of type $c$, according to (9), we shall have

$$
v_{i c}(t+\Delta t)=v_{i c}(t)+M\left\{\Delta V_{i}^{(c)}(t, \Delta t)\right\}=v_{i c}(t)+f(i, c, t) \Delta t+o(\Delta t),
$$


and passing to the limit $\Delta t \rightarrow 0$, we obtain first order inhomogeneous ordinary DE (ODE) $\frac{d v_{i c}(t)}{d t}=f(i, c, t)$. Therefore, finally, the expected income of the system $S_{i}$ will be equal to

$$
\begin{aligned}
v_{i}(t) & =v_{i 0}+\sum_{c=1}^{r} v_{i c}(t)=v_{i 0}+\sum_{c=1}^{r} \alpha_{i}^{(c)}\left(\lambda\left(p_{0 c i c}-1\right)+1\right) t+ \\
+ & \sum_{c=1}^{r}\left[\mu_{i c}\left(\alpha_{i}^{(c)} p_{i c 0 c}-\sum_{j=1}^{n} \sum_{s=1}^{r} \varphi_{i j}^{(c)} p_{i c j s}\right)_{t_{0}}^{t} \int_{i c}(\tau) d \tau-\right. \\
- & \left.\sum_{i=1}^{n} \sum_{j=0}^{n} \sum_{c, s=1}^{r} \mu_{i c} p_{i c j s} \int_{t_{0}}^{t} \rho_{i c}(\tau) d \tau\right], i=\overline{1, n} .
\end{aligned}
$$

\section{Finding the mean number of busy lines in network systems}

Let $N_{i c}(t)$ be the mean number of customers of type $c$ in the system $S_{i}$ at time $t$, $i=\overline{1, n}, c=\overline{1, r}$. For finding $N_{i c}(t)$ we can can apply the MVA method. It is used to find the mean number of customers another technique.

As far as going to the network there constantly arrive Poisson process of rate $\lambda$, i.e. the probability of arrivals of customers of type $c$ to the QS $S_{i}$ during time $\Delta t$ has the form $P_{a c}(\Delta t)=\frac{\left(\lambda p_{0 c i c} \Delta t\right)^{a}}{a !} e^{-\lambda p_{0 i c} \Delta t}, c=\overline{1, r}, l=0,1,2, \ldots$, then the mean number of customers of type $c$, arrived from the outside to the QS $S_{i}$ during the time $\Delta t$ equals $\lambda p_{0 c i c} \Delta t, c=\overline{1, r}$. Obviously, that $\mu_{i c} \rho_{i c}(t) \Delta t$ - means the number of customers of type $c$, that have departed the QS $S_{i}$ during time $\Delta t$, a $\sum_{\substack{j=1 \\ j \neq i}}^{n} \sum_{s=1}^{r} \mu_{j s} p_{j s i c} \rho_{j s}(t) \Delta t-$ the mean number of customers of type $c$, that have arrived the QS $S_{i}$ from other QS during time $\Delta t, c=\overline{1, r}$. Therefore

$$
\begin{gathered}
N_{i c}(t+\Delta t)-N_{i c}(t)= \\
=\lambda p_{0 c i c} \Delta t+\sum_{\substack{j=1 \\
j \neq i}}^{n} \sum_{s=1}^{r} \mu_{j s} p_{j s i c} \rho_{j s}(t) \Delta t-\mu_{i c} \rho_{i c}(t) \Delta t, i=\overline{1, n}, c=\overline{1, r},
\end{gathered}
$$

where if $\Delta t \rightarrow 0$ follows ODE system for $N_{i c}(t)$ : 


$$
\frac{d N_{i c}(t)}{d t}=\sum_{\substack{j=1 \\ j \neq i}}^{n} \sum_{s=1}^{r} \mu_{j s} p_{j s i c} \rho_{j s}(t)-\mu_{i c} \rho_{i c}(t)+\lambda p_{0 c i c}, i=\overline{1, n}, c=\overline{1, r}
$$

It is impossible to find exactly the value $\rho_{i c}(t)$ and therefore we approximate it by the expression

$$
\rho_{i c}(t)=\left\{\begin{array}{c}
N_{i c}(t), N_{i c}(t) \leq m_{i}, \\
m_{i}, N_{i c}(t)>m_{i},
\end{array}=\min \left(N_{i c}(t), m_{i}\right) .\right.
$$

Then the system of equations for (12) takes the form

$$
\begin{gathered}
\frac{d N_{i c}(t)}{d t}=\sum_{\substack{j=1 \\
j \neq i}}^{n} \sum_{s=1}^{r} \mu_{j s} p_{j s i c} \min \left(N_{j s}(t), m_{j}\right)- \\
-\mu_{i c} \min \left(N_{i c}(t), m_{i}\right)+\lambda p_{0 c i c}, i=\overline{1, n}, c=\overline{1, r} .
\end{gathered}
$$

That is a system of inhomogeneous linear ODE with discontinuous right-hand sides. It should be solved by dividing the phase space into a number of areas and finding solutions to each of them. The system (14) can be solved, for example, using the tools of computer mathematics Maple. Set initial conditions $v_{i c}(0)=v_{i c 0}$, $i=\overline{1, n}, c=\overline{1, r}$, we can find the expected incomes of network systems.

If the network operates so that there are no observed queues in the average (a lowtraffic regime), i.e. $\min \left(N_{i c}(t), m_{i}\right)=N_{i c}(t), i=\overline{1, n}, c=\overline{1, r}$, then the system (14) takes the form:

$$
\frac{d N_{i c}(t)}{d t}=\sum_{\substack{j=1 \\ j \neq i}}^{n} \sum_{s=1}^{r} \mu_{j s} p_{j s i c} N_{j s}(t)-\mu_{i c} N_{i c}(t)+\lambda p_{0 c i c}, i=\overline{1, n}, c=\overline{1, r},
$$

The system (15) can be rewritten in the matrix form

$$
\frac{d N(t)}{d t}=Q N(t)+f
$$

where $N^{T}(t)=\left(N_{1 c}(t), N_{2 c}(t), \ldots, N_{n c}(t)\right), c=\overline{1, r}, Q=\|q\|_{n \times n}$ - matrix, consist of elements $q_{i j}=\sum_{s=1}^{r} \mu_{j s} p_{j s i c}$, if suppose that the probabilities $p_{i c i c}$ equal $-1, i, j=\overline{1, n}$ , $f$ - column vector, with elements $\lambda p_{0 c i c}, i=\overline{1, n}$. Solution of the system (16) has the form 


$$
N(t)=N(0) e^{Q t}+f \int_{0}^{t} e^{Q(t-\tau)} d \tau,
$$

where $N(0)$ - some given initial conditions, $e^{Q t}$ - the matrix exponent.

\subsection{Example}

Consider a network as described above. We consider the case when the network is functioning so that there are no observed queues in the average. QS count equals $n=9$, input rate equals $\lambda=10$, count of type customers equals $r=6$. Service rates equal: $\mu_{11}=0.5, \mu_{22}=1, \mu_{33}=0.3, \mu_{44}=2.5, \mu_{51}=0.33, \mu_{62}=2, \mu_{75}=0.1$, $\mu_{76}=0.2, \mu_{86}=0.2, \mu_{91}=1, \mu_{92}=0.55, \mu_{93}=0.05, \mu_{94}=1$, other service rates equal zero, because according to the model described above in each QS do not service all types of customers. Probabilities transitions: $p_{0191}=1, p_{0292}=1, p_{0494}=1$, $p_{1151}=0.2, \quad p_{1191}=0.2, \quad p_{1192}=0.2, \quad p_{1193}=0.2, \quad p_{1194}=0.2, \quad p_{2262}=0.2$, $p_{2291}=0.2, p_{2292}=0.2, p_{2293}=0.2, p_{2294}=0.2, p_{3375}=\frac{1}{6}, p_{3386}=\frac{1}{6}, p_{3391}=\frac{1}{6}$, $p_{3392}=\frac{1}{6}, \quad p_{3393}=\frac{1}{6}, \quad p_{3394}=\frac{1}{6}, \quad p_{4491}=0.25, \quad p_{4492}=0.25, \quad p_{4493}=0.25$, $p_{4494}=0.25, \quad p_{5111}=1, \quad p_{6222}=1, \quad p_{7533}=1, \quad p_{7633}=1, \quad p_{8676}=1, \quad p_{9111}=0.5$, $p_{9222}=0.5, p_{9333}=0.5, p_{9444}=0.5, p_{9303}=0.5, p_{9404}=0.5$, other probabilities equal zero, because of the network structure and the model described above - other transitions are impossible.

Consider the time period of 10 hours, $t \in[0, T], T=24$. Then using formulas for finding the mean number of customers, in the package "Mathematica" numerical solutions were obtained for the mean number of customers of type $c$ and the expected incomes of the network QS and charts of incomes of these queueing systems, see Figure $2\left(N_{11}(0)=100, N_{86}(0)=80, N_{912}(0)=0\right)$.

The vector of server-queues has the form $m=\left(m_{1}, m_{2}, \ldots, m_{n}\right)=$ $=(17,37,15,5,1510,25,15,1005,15)$. The random incomes have the form: $R_{15}\left(\xi_{11}\right)=1000 \xi_{11}, \quad R_{19}\left(\xi_{11}\right)=500 \xi_{11}, \quad R_{26}\left(\xi_{22}\right)=3000 \xi_{22}, \quad R_{29}\left(\xi_{22}\right)=1000 \xi_{22}$, $R_{51}\left(\xi_{51}\right)=100 \xi_{51}, R_{62}\left(\xi_{62}\right)=1000 \xi_{62}, R_{91}\left(\xi_{91}\right)=100 \xi_{91}, R_{92}\left(\xi_{92}\right)=10 \xi_{92}$. Expectations: $M\left\{\eta_{i c}\right\}=\alpha_{i}^{(c)}, M\left\{r_{i c}\right\}=\gamma_{i}^{(c)}, i=\overline{1, n}, c=\overline{1, r}$, respectively equal: $\alpha_{3}^{(3)}=5000$, $\alpha_{4}^{(4)}=500, \quad \alpha_{7}^{(5)}=400, \quad \alpha_{7}^{(6)}=200, \quad \alpha_{8}^{(6)}=100, \quad \alpha_{9}^{(3)}=\alpha_{9}^{(4)}=200, \quad \gamma_{3}^{(3)}=1000$, $\gamma_{4}^{(4)}=14000, \gamma_{7}^{(5)}=\gamma_{7}^{(6)}=100, \gamma_{8}^{(6)}=500, \gamma_{9}^{(3)}=2500, \gamma_{9}^{(4)}=800$. Let at the initial time $t_{0}=0$ system incomes be equal to zero. Then, using formula (11) and the math packet Wolfram Mathematica, expressions for the income changes of the 
QS $S_{i}$ and customers of type $c, i=\overline{1, n}, c=\overline{1, r}$, have been obtained. The chart of the expected income, for example for the QS $S_{9}$, is shown in Figure 3.

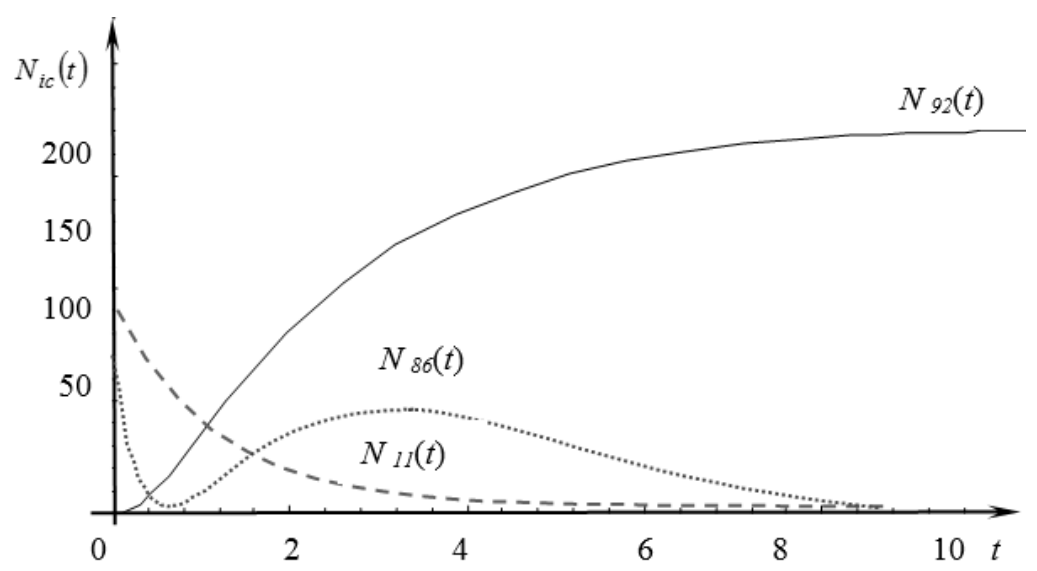

Fig. 2. Changes of mean number of customers

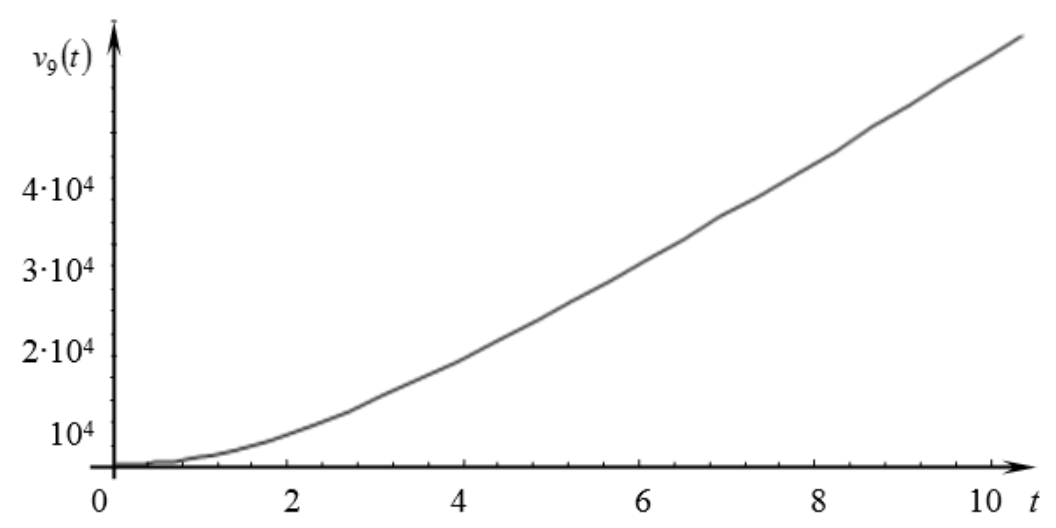

Fig. 3. Total income changes of the QS $\mathrm{S}_{9}$ for four types of customers

\section{Conclusions}

In the paper Markov HM-network with FIFO disciplines has been studied, when incomes from the state transition are RV with given mean values. Such incomes are dependent on the time of customer service in the network. Approximate expressions for the expected incomes QS of the network have been obtained. The method of finding the mean number of customers in the network systems has also been described.

A method of finding the expected incomes in the systems based on the use of found approximate and exact expressions for the mean values of the random 
incomes for an open HM-network with different types of customers and manyserver queues has been also developed.

The obtained results can be used in modeling income changes in various INS.

\section{References}

[1] Matalycki M., Pankow A., Research of Markov queueing network with central system and incomes, Computer Science 2004, 4(7), 23-32.

[2] Naumenko V., Research of model changes of incomes of the item for collective use, Vestnik of GrSU. A Series of Mathematics, Physics, Computer Science, Computer Facilities and Management 2012, 1, 143-157. 